\title{
DIAGNOSTIC AND THERAPEUTIC DIFFICULTIES FOR THE ENT SURGEON IN MALIGNANT TUMOURS OF THE NECK
}

\author{
Mihail TUSALIU1,2®, Iulia TITA ${ }^{1}$, Anca CIOBOTARIU ${ }^{1}$, Gelu GROSU ${ }^{1}$, Diana TUAS ${ }^{1}$, \\ Ruxandra RANETE ${ }^{1}$
}

1 „Prof. Dr. D. Hociota“ Institute of Phonoaudiology and Functional ENT Surgery, Bucharest, Romania

${ }^{2}$ „Carol Davila“ University of Medicine and Pharmacy, Bucharest, Romania

Received 26 Apr 2020, Accepted 14 May 2020

hitps://doi.org/10.31688/ABMU.2020.55.2.15

\begin{abstract}
Despite the remarkable technological advances of recent decades, there is not a general consensus or universal standard in the diagnosis and therapeutic management of malignant tumours of the neck. Beginning by defining the neck as the anatomical-surgical region with many organs and a series of very important vascular and nervous structures, the multitude and complexity of pathology elements that could be encountered at this level make cancers of the neck often difficult to diagnose and treat. The authors describe and synthesize the main types of cervical malignancies that the ENT surgeon may encounter in current medical practice, emphasizing the particularities of each individual pathological entity, as well as the diagnostic and therapeutic problems it may pose.
\end{abstract}

Keywords: malignant tumours, neck, ENT surgery.

\section{List of abbreviations}

C6 $-6^{\text {th }}$ cervical vertebra

CT - computed tomography

\section{Résumé}

Difficultés diagnostiques et thérapeutiques pour les chirurgiens ORL concernant les tumeurs malignes du cou

Malgré les avancées technologiques remarquables de ces dernières décennies, il n'existe pas de consensus général ou de norme en ce qui concerne le diagnostic et le traitement des tumeurs malignes du cou. $\grave{A}$ partir de la définition du cou comme une région anatomique chirurgicale, avec un groupe d'organes et de structures vasculaires et nerveuses très importants et nombreux, la multitude et la complexité des éléments pathologiques qu'on peut découvrir à ce niveau-là font souvent de ce type de tumeurs des entités difficiles à diagnostiquer et à traiter. Les auteurs essaient de décrire et de synthétiser les catégories principales de tumeurs malignes cervicales que le chirurgien ORL peut rencontrer dans sa pratique habituelle, en se basant sur les particularités de chaque pathologie, tout en tenant compte des problèmes diagnostiques et de conduite thérapeutique soulevés par celles-ci 
EBV - Epstein-Barr virus

ENT - otorhinolaryngology or ear, nose, throat

$\mathrm{G} 2-2^{\text {nd }}$ differentiation grade cancer cells

$\mathrm{G} 3-3^{\text {rd }}$ differentiation grade cancer cells

IHC - immunohistochemistry

MRI - magnetic resonance imaging

PET-CT - positron emission tomography-computed tomography

\section{INTRODUCTION}

Neck is a topographic region between the head and the thorax, an anatomical-surgical region with a high complexity, sheltering a series of organs, as well as important vascular and nervous structures (Fig. 1). Therefore, in this high-complex area, a multitude of pathology elements can be encountered, which continue to pose many problems of diagnosis and treatment ${ }^{1}$.

Most malignant tumours located in the neck area are metastatic adenopathies. This implies the development of squamous cell carcinoma metastases in various lymph nodes in the head and neck area, from a primary tumour located in the upper digestive tract. In addition to metastatic adenopathies, there are a number of primary malignancies of the neck, including: Hodgkin's or non-Hodgkin's lymphomas, sarcomas, thyroid or parathyroid gland carcinomas, salivary gland malignancies, benign tumours that had undergone a malignant transformation (branchial cleft cyst, thyroglossal duct cyst), malignant vascular tumours. Also, in the neck area, distant lymph node metastases may occur from primary tumours
Mots-clés: tumeurs malignes, cou, chirurgie ORL.

originating from organs such as lung, kidney, prostate, stomach, breast ${ }^{1-4}$.

Drawing from Rouvière's classical, exhaustive work, describing groups of ganglia of the head and neck, in terms of cervical topographic anatomy, six regions of particular importance in current medical practice can be distinguished ${ }^{5}$.

\section{Carcinoma of unknown Primary origin}

Cervical metastatic malignancy with unknown primary origin remains one of the biggest challenges, both in terms of diagnosis and therapeutic management. It is characterized by the presence of one or several carcinoid metastases of the lymph nodes, as confirmed by anatomopathological examination, without identifying the primary tumour. In about $25 \%$ of the cases, it is found while undergoing treatment for metastatic adenopathy, while in other cases it may be identified post-mortem or never be discovered at all ${ }^{6-7}$.

Spinocellular carcinoma is the most frequently encountered histological type, found in up to $75 \%$ of cases. Other histological types, such as

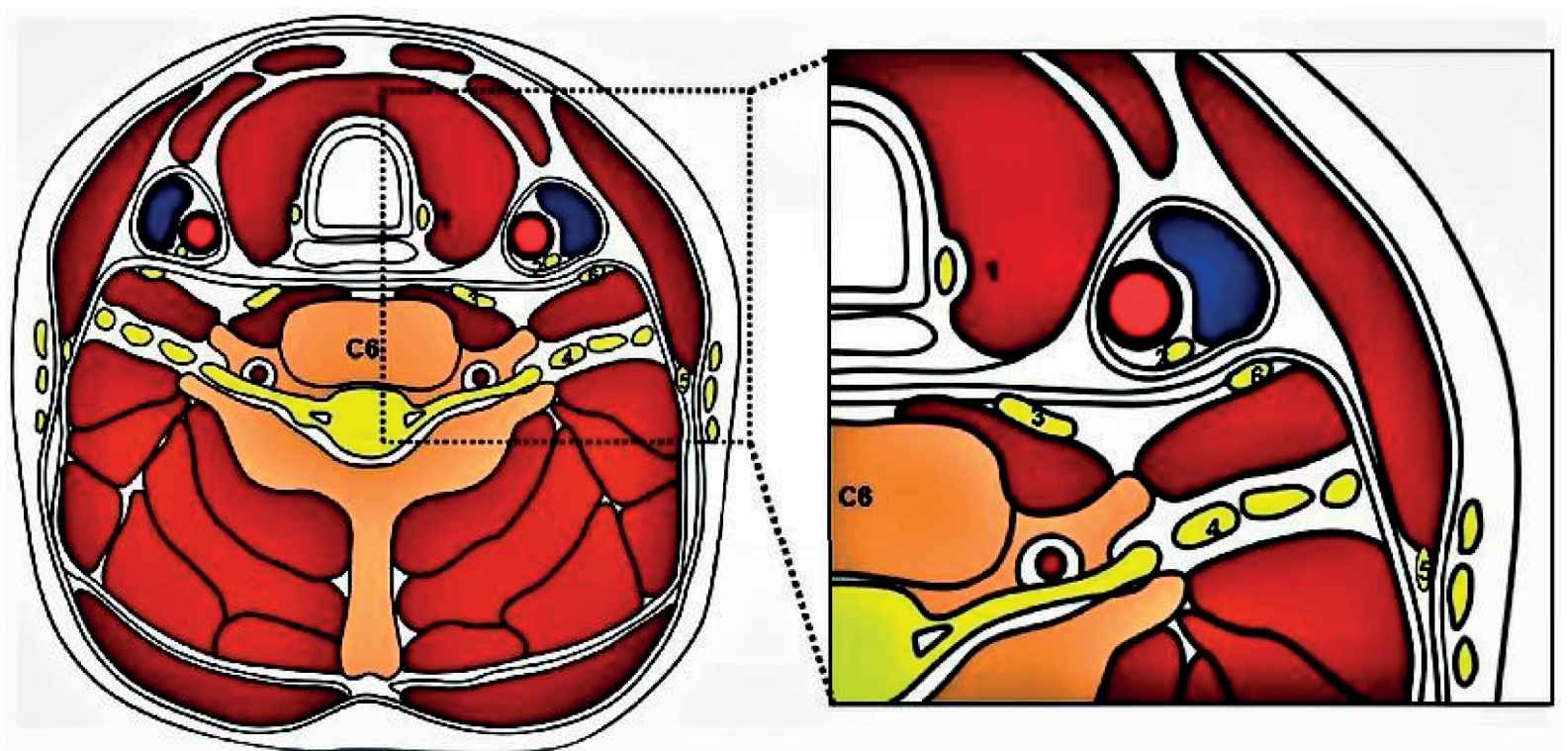

Fig. 1. Transverse section at the level of C6 vertebra. 
undifferentiated carcinoma, adenocarcinoma and melanoma, are less frequent ${ }^{8}$.

About $10 \%$ of lymph nodes metastases originate from unknown primary tumours. In about a third of cases, the primary tumour is discovered during the course of the patient's lifetime, with up to $70 \%$ of these primary tumours being subject to the ENT area of expertise, situated in the head and neck region (nasopharynx, oral cavity, hypopharynx, larynx, thyroid gland). In $20-30 \%$ of cases, the primary site is located within the lungs, gastrointestinal tract, breasts or kidneys ${ }^{9-11}$.

The diagnosis must consider two aspects: the determination of the exact histological type of the cervical mass and the origin of the primary tumour. In addition to the ENT clinical examination, a rigid and flexible pharyngo-laryngeal endoscopy must be carried out, focusing on increased risk areas, or on so-called hidden anatomical areas (lateral nasopharyngeal wall, amygdala region, hypopharynx ${ }^{12}$. Biopsy fragments are harvested from all suspicious areas. An ultrasound of the cervical region is performed, as well as imaging techniques (computed tomography - CT, magnetic resonance imaging - MRI),

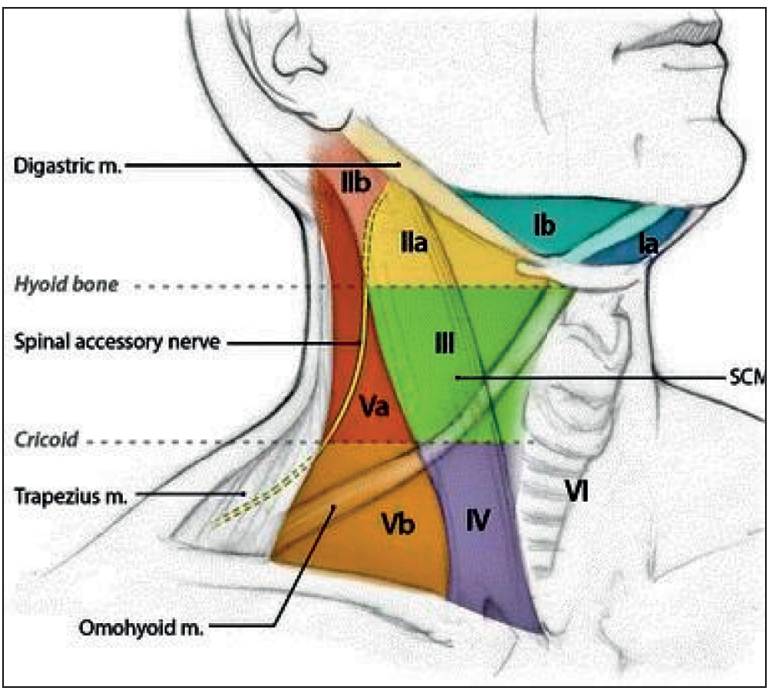

Fig. 2. Lymphatic node groups of head and neck. that play a role in the evaluation of cervical masses and the diagnosis of the primary tumour ${ }^{13-16}$.

If the primary tumour cannot be identified, the diagnosis of metastatic adenopathy is established by performing an ultrasound-guided fine needle biopsy ${ }^{17}$ or by carrying out surgical exploration of the cervical tumours with total lymph node excision and anatomical-pathological examination ${ }^{8}$.

We illustrate the above with a clinical case of a 58-year-old male, admitted for a right latero-cervical mass, cervical pain and right reflex otalgia. During the surgical intervention, a solid giant cervical tumour is found, measuring $9 / 5 / 4 \mathrm{~cm}$, inhomogeneous, that infiltrates the adjacent tissues. A systematic, step by step, dissection was performed. The tumour infiltrated the superior half of the right sternocleidomastoid muscle, right internal jugular vein in the cervical segment and the right superior thyroid artery. The tumour penetrated the right parapharyngeal space and was in contact with the tip of the right mastoid process and the vertebral plane. The tumour was laboriously detached from these structures. The right spinal, vagus and hypoglossal nerves were identified and dissected. The right internal jugular vein was identified and ligated at the superior and inferior cervical poles. The tumour was detached from the carotid sinus, with dissection of the adventitia of the right common carotid artery and the right internal carotid artery. The right superior thyroid artery emerged from the carotid sinus, entered the tumour and was ligated. The tumour was excised within macroscopic limits, together with the right internal jugular vein. The excised material was referred to histopathologic examination. A tissue sample was harvested separately from the adventitia of the common carotid artery as safety margin and was referred to histopathologic examination. The histopathologic exam showed lymph nodes with G2 moderately differentiated spinocellular epidermoid carcinoma metastasis. The adventitial fragment from the common carotid artery had a normal histologic aspect.

The clinical case detailed here illustrates the various difficulties encountered in the diagnosis and therapeutic management of this pathology. Any

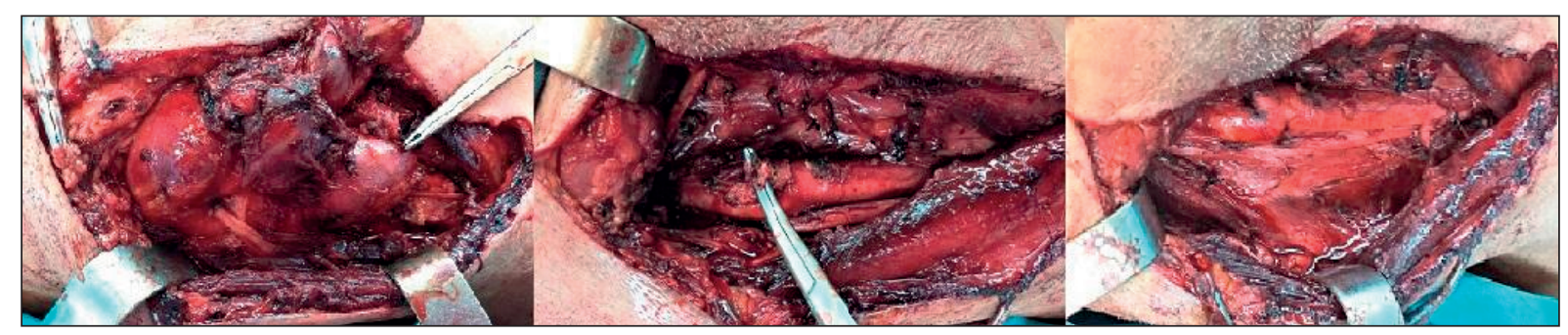

Fig. 3. Intraoperative view - metastatic cervical adenopathy of unknown primary site. 
condition in its early stage may be difficult to identify, due to the lack of symptoms. The patient did not show clinical manifestations suggesting a neoplastic disease before the cervical lymph nodes started swelling. This delay, in turn, influences the subsequent progression of the disease and the case prognosis.

All efforts should be made in trying to identify the primary tumour. A flexible and rigid pan endoscopy of the upper aerodigestive tract, under general anesthesia, harvesting biopsies from lesions or suspected areas, must be considered. Rigid esophagoscopy, upper digestive tract endoscopy, tracheobronchoscopy and bronchoalveolar lavage are performed. A whole body CT scan or MRI (head, neck, thorax, abdomen) could be useful to identify the origin of the primary tumour. These imaging investigations should be supplemented by a positron emission computed tomography (PET-CT) scan. PET-CT can be used to diagnose carcinoma of unknown primary origin, for lymph nodes metastases evaluation, identification of distant metastases or synchronous cancer identification. In approximately $25 \%$ of cases, PET-CT can detect primary tumours otherwise undetectable by other diagnostic means. In about $20 \%$ of cases, within a timeframe of up to two years, the occurrence of lymph node metastases precedes the appearance of the primary tumours, therefore very rigorous, active outpatient care is required, as well as the adenopathies' histopathological type identification in the quest to find the origin of the tumour ${ }^{2,15}$.

Serology testing for EBV antibodies must be considered as well, given the virus' involvement in the etiology of nasopharyngeal cancer ${ }^{18}$.

While further carrying out the surgical exploration of the cervical area for diagnosis purposes, if an extemporaneous histopathological examination confirms a metastatic carcinoma, lymph nodes removal within the same surgery is recommended, usually followed by radiotherapy. The therapeutic protocol of these tumours of unknown primary origin is not standardized, with ongoing discussions carried out in the medical literature ${ }^{19}$.

When operating a malignant tumour, Halsted's principles of oncological surgery must be considered: „in block“ resection reaching healthy tissue, negative tumour margins, lymph node removal for regional disease control, histopathological examination +/. immunohistochemical tests, surgical resection of isolated metastases, when these are technically feasible ${ }^{20}$.

Despite remarkable technological advances in diagnosis techniques, cervical metastatic adenopathy of unknown primary origin represents a great challenge. Difficulties in identifying or recognizing its origin automatically translate into inadequate or incomplete treatment. Therefore, further efforts must be invested for both diagnostic (molecular, genetic, imaging) and therapeutic purposes, in order to increase patient's survival.

\section{Malignancies of the parapharyngeal space}

Tumours that arise in this region raise a series of problems regarding symptomatology, growth rate and evolution over time, benign or malignant nature, therapeutic approach in general and surgical approach. Although these primary malignancies of the parapharyngeal space are rare, the specific anatomy of this area makes their access difficult for clinical evaluation. Therefore, neoplasms arising out of this region may represent a peculiar challenge for surgeons $\mathrm{s}^{21}$.

Although most of the parapharyngeal space tumours are benign, a small number of malignant tumours can also be encountered. Most of these malignant tumours originate from the salivary glands. These malignancies can be asymptomatic for a long time. Considering the deep location of the parapharyngeal space, early detection of a pathological process may be very difficult ${ }^{22}$. Thus, a thorough investigation of the patient plays an important role in diagnosis and in developing an appropriate therapeutic plan. Imaging techniques (ultrasound, CT, MRI) play an essential part both in the visualization of the tumour, as well as in elaborating a therapeutic plan, by locating the tumour in either the pre-styloid or post-styloid space, establishing the interaction with the parotid gland and anatomical risk elements posed by surgery, determining the tumour margins, and establishing its extension and degree of invasion of adjacent tissues ${ }^{23,24}$

Such a tumour of the left parapharyngeal space was observed in the clinical case of a 22-year-old patient admitted to our clinic for a left retromandibular and retro auricular swelling, lockjaw, intermittent left hemicrania and of left aural fullness. The MRI examination described an infiltrative tumour growth, centered on the left upper parapharyngeal space, coming in posterolateral contact with the left long neck muscle, and laterally with the left pterygoid muscles; it is in contact with the mandible in the vicinity of the temporomandibular joint, laterally and posteriorly it engulfs the left internal carotid artery in the cervical segment and comes into direct contact with the internal jugular vein; the growth has no endocranial extension and is accompanied by bilateral latero-cervical adenopathies.

Surgery was performed, revealing solid irregular, non-homogeneous tumour with multiple adhesions and infiltrating the adjacent structures. Dissection of the tumour was carried out with difficulty, ligation and sectioning of the left internal jugular vein, which was infiltrated by the tumour. 


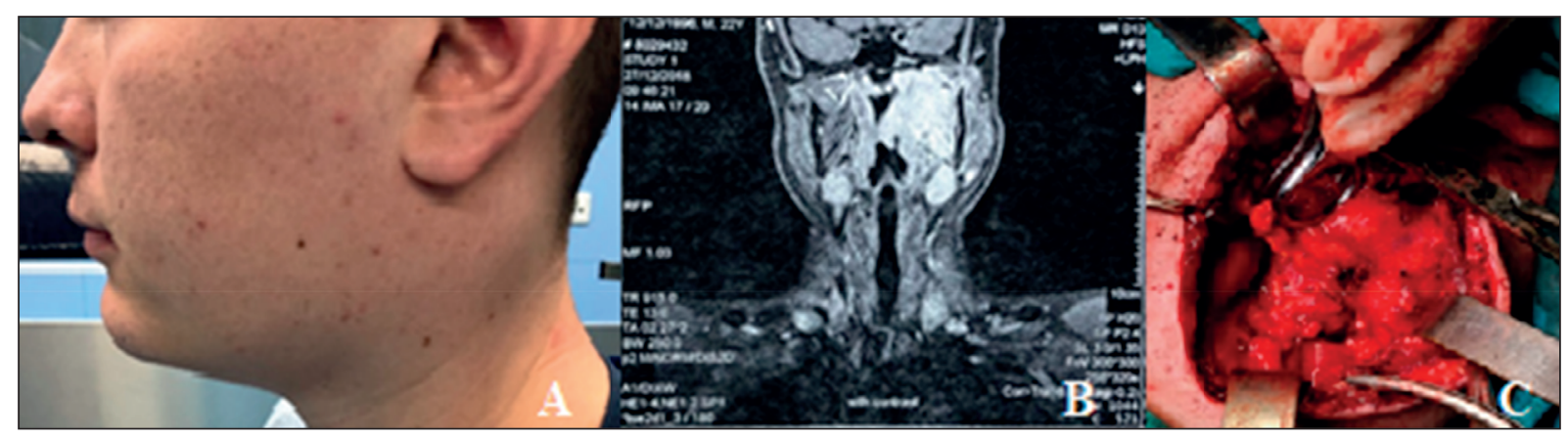

Fig. 4. A - Left parapharyngeal mass. B - CT scan aspect. C - Intraoperative view.

Dissection was continued. The growth was found to be in close contact with the wall of the left internal carotid artery; detachment from the carotid artery adventitia was performed with difficulty. The digastric muscle was cut, revealing that the entire left parapharyngeal space was occupied by the tumour; the tumour was detached with difficulty from the sternocleidomastoid and scalene muscles, the inferior border of the mastoid process, the base of the skull and the first cervical vertebrae; complete resection within macroscopic limits of the tumour in block with the left internal jugular vein, ablation of peritumoural jugular-carotid and spinal lymph nodes were performed.

Histopathological examination revealed a left parapharyngeal G3 poorly differentiated carcinoma, and lymph nodes with G3 poorly differentiated carcinoma metastases, the immunohistochemical examination supporting this diagnosis.

The surgical intervention detailed here was mainly meant to collect multiple biopsy fragments in order to establish a diagnosis, and at the same time, to surgically resect the tumour mass to the extent that this was feasible. The difficulty posed by the case consisted both in choosing the optimal surgical approach, as well as in surgery itself, considering the difficult access and concern for the radical excision of the tumour, while preserving the functionality of the vascular, nervous and osseous locoregional structures (carotid artery and its bifurcations, cranial nerves VII, IX, X, XI, XII, left branch of the mandible).

In the clinical case detailed here, the surgical treatment resulted in complete removal of the tumour, without major intraoperative complications, while short-term postoperative complications and functional and aesthetic sequelae were minimal and generally reversible.

\section{LYMPHOMAS OF THE CERVICAL AREA}

Another category of tumours located in the neck area are lymphomas. With a steadily increasing incidence observed in recent decades, together with leukemia, they represent $10-15 \%$ of malignancies and the second most frequent type of head and neck cancer ${ }^{25}$.

Diagnosis and treatment of this pathology still raise many difficulties. Cervical lymphadenopathy is a serious diagnostic problem, as it occurs in a wide variety of acute and chronic conditions. There is a long asymptomatic timeframe in the evolution of the disease, with swelling of the superficial lymph nodes being usually accidentally discovered, either by patients themselves, or by doctors during routine examinations. By the time patients come to the doctor, most are in an advanced stage of the disease (III or IV Ann Arbor) ${ }^{26,27}$.

Through surgical intervention, tissue is collected for histopathological examination, thus establishing a diagnosis of certainty. The detailed anatomopathological examination using immunohistochemistry (IHC) and cytogenetic techniques allows the correct classification of the type of lymphoma. It is therefore possible to establish a correct and adequate course of treatment, that will increase survival and improve prognosis ${ }^{18}$.

In malignant lymphomas, the positive diagnosis is established only through an entire examination of anatomopathological biopsy. Tissue fragments can be obtained by fine-needle biopsy or excisional biopsy. Fine needle biopsy differentiates an inflammatory lesion from a carcinoma, but it can only provide oriented lymphoma diagnosis, on its own being insufficient to differentiate between Hodgkin's and non-Hodgkin's lymphomas ${ }^{28}$. Data on tumour immunohistochemistry, cytogenetic data and special markers can be obtained only by excisional biopsy. It represents the „gold-standard“ method of diagnosis for all malignant lymphomas. For accuracy of diagnosis, size of sample and careful manipulation of harvested tissue are crucial. Diagnosis, however, is not established following node fragment biopsy, but only after the extracapsular excision of an entire cervical lymph node for an extensive analysis of peripheral 
tissue. Together with the IHC and cytogenetic examinations, all markers of differentiation and prognosis that lead to a correct and complete lymphoma diagnosis are identified. The next stage in the diagnosis of malignant lymphoma is carried out by the hematologist, who will perform specific tests to determine the stage of the disease ${ }^{29}$.

Although in cervical lymphomas the purpose of surgical treatment is, par excellence, a diagnostic one, there are clinical situations when surgery plays other roles in the management of malignant lymphopathies. When encountering voluminous latero-cervical tumour masses, with or without a respiratory axis or pharyngo-esophageal vasculo-nervous compression syndrome, it is possible to perform cytoreductive surgery procedure, which significantly reduces the volume of the tumour ${ }^{30}$. Surgery, in this case, plays a role in enhancing the immune response, the effect of chemotherapy and radiotherapy and last but not least it plays a psychological role for the patient. Therapeutic difficulties may also be encountered in emergency surgery, such as a large thyroid lymphoma, which can lead to acute respiratory failure through extrinsic compression of the respiratory axis. Thus, surgery may play an assisting role when vital functions are impaired. In this case, a tracheostomy is indicated, but not always easy to perform ${ }^{18}$.

In conclusion, considering difficulties encountered and the efforts made for correct diagnosis and the appropriate therapy for malignant lymphoma, the complex multidisciplinary approach, clinical integration, morphological and cytogenetic elements, collaboration and combined expertise of the pathologist, hematologist, oncologist, radiotherapist, radiologist, nutritionist, psychologist, together make up the most well established method of approaching a patient with lymphoma ${ }^{31}$.

\section{Other cervical malignancies}

Thyroid neoplasms constitute a significant number of cervical malignancies. In addition to the clinical and imaging evaluations, fine needle aspiration cytology must be considered a mandatory integral part of diagnosis procedures ${ }^{16}$. If cytology is positive for cancer, surgical treatment is indicated. Surgery is often the main therapeutic means in thyroid malignancies, total thyroidectomy being the most common surgical intervention performed. Surgical management involves careful, meticulous and rigorous dissection, so that the recurrent nerves can be identified and preserved, when they are not invaded by neoplastic processes. The superior laryngeal nerves and parathyroid glands should also be protected and preserved. An important immediate postoperative complication is upper airway obstruction, due to laryngeal edema or surgical site hematoma ${ }^{32}$. This requires prompt intervention (reintubation, wound opening and hematoma evacuation) to restore normal airflow to the laryngotracheal axis. Another difficulty encountered in thyroid malignancy is posed by large tumours, exercising an extrinsic compression on the airway, leading to acute respiratory failure, with or without moving it from the midline, in which case tracheostomy would be technically difficult to perform (as well as being conditioned by time pressure).

Malignant tumours of the salivary glands also pose issues in terms of diagnosis or therapeutic course of action. The great diversity of pathology elements makes the use of fine needle aspiration biopsy techniques in establishing the diagnosis a real challenge, even for an experienced pathologist ${ }^{33}$.

Surgical treatment is the main therapeutic means used in malignancies of the salivary glands. In parotid malignancies, during the various parotidectomy surgical procedures performed, the main element of difficulty is posed by the management of the facial nerve. It should be preserved if its functionality was intact before surgery, and partially or totally sacrificed, only if this facilitates the complete resection of the tumour. It is useful in this regard to use a facial nerve monitor or stimulator intraoperatively. If the nerve is partially damaged, its continuity must be restored, using the greater auricular nerve $\mathrm{e}^{34}$.

In malignancies of the submaxillary gland, preservation of the facial, lingual and hypoglossal nerves branches must be attempted. Also, it is important that the resection be extensive, extracapsular, doubled by selective lymph node removal for ganglion groups I, II and III, in order to reduce the risk of residual tumour tissue and relapse $\mathrm{e}^{35}$.

Other cervical malignancies that may raise diagnostic or therapeutic management problems are:

- nerve tumours, generally benign, but with the possibility of malignant transformation ;

- diverse histological subtypes of sarcoma;

- vascular tumours such as angiosarcoma, Kaposi's sarcoma or hemangiopericytoma ${ }^{16}$.

\section{Conclusions}

Beginning by defining the neck as an anatomical-surgical region with many organs and a series of very important vascular and nervous structures, the multitude and complexity of pathology elements that may be encountered in this level make cancers of the neck often difficult to diagnose and treat. In this regard, further efforts must be made to understand the neoplastic process, in general, and the clinical situations we may encounter in particular, so that by 
synthesizing all obtained theoretical and practical data to approach a personalized therapy, where each subtype is treated as a separate disease.

Given the difficulties encountered and the efforts made for the correct diagnosis and appropriate therapy of a malignant tumour of the neck, the complex multidisciplinary approach integrating morphological, cytogenetic, chemical, paraclinical and therapeutic elements, represents the best method of approaching a patient with such a pathology.

\section{Author Contributions:}

M.T. conceived the original draft preparation. I.T., D.T. and R.R. were responsible for conception and design of the review. M.T., A.C. and G.G. were responsible for the data acquisition. M.T. and I.T. were responsible for the collection and assembly of the articles/published data, and their inclusion and interpretation in this review. All authors contributed to the critical revision of the manuscript for valuable intellectual content. All authors have read and agreed to the published version of the manuscript.

\section{Compliance with Ethics Requirements:}

„The authors declare no conflict of interest regarding this article"

„The authors declare that all the procedures and experiments of this study respect the ethical standards in the Helsinki Declaration of 1975, as revised in 2008(5), as well as the national law. Informed consent was obtained from all the patients included in the study"

"No funding for this study"

\section{Acknowledgements:}

None

\section{References}

1. Müller von der Grün J, Tahtali A, Ghanaati S, Rödel C, Balermpas P. Diagnostic and treatment modalities for patients with cervical lymph node metastases of unknown primary site - current status and challenges. Radiat Oncol. 2017;12(1):82.

2. Wang Y, He SS, Bao Y, et al. Cervical lymph node carcinoma metastasis from unknown primary site: a retrospective analysis of 154 patients. Cancer Med. 2018;7(5):1852-9.

3. Zhuang SM, Wu X, Li J, Zhang G. Management of lymph node metastases from an unknown primary site to the head and neck. Mol Clin Oncol. 2014;2(6):917-22.

4. Ginghina $\mathrm{O}$, Negrei C, Hudita A, et al. In vitro impact of some natural compounds on HT-29 colorectal adenocarcinoma cells. Farmacia. 2017;65(6):947-953.

5. Lin HW, Roberts DS, Harris JP. Cummings review of otolaryngology. Elsevier Health Sciences. 2016:92-94.

6. Waltonen JD, Ozer E, Hall NC, Schuller DE, Agrawal A. Metastatic carcinoma of the neck of unknown primary origin: evolution and efficacy of the modern workup. Arch Otolaryngol Neck Surg. 2009;135(10):1024-9.

7. Petrakis D, Pentheroudakis G, Voulgaris E, Pavlidis N. Prognostication in cancer of unknown primary (CUP): development of a prognostic algorithm in 311 cases and review of the literature. Cancer Treat Rev. 2013;39(7):701-8.

8. Pavlidis N, Pentheroudakis G. Cancer of unknown primary site. Lancet. 2012;379(9824):1428-35.

9. Nagarkar R, Wagh A, Kokane G, Roy S, Vanjari S. Cervical lymph nodes: a hotbed for metastasis in malignancy. Indian J Otolaryngol Head Neck Surg. 2019;71(1):976-80.

10. Socea B, Nica AA, Bratu O, et al. Incidental finding of a sigmoid intussusception associated with rectal prolapse - a case report. Arch Balk Med Union. 2018;53(1):143-146.

11. Diaconu CC, Arsene D, Balaceanu A, Bartos D. A rare tumor revealed by abdominal trauma: case presentation. Romanian Journal of Morphology and Embryology 2014;55(3):973-976.

12. Arrangoiz R, Galloway TJ, Papavasiliou P, Ridge JA, Lango MN. Metastatic cervical carcinoma from an unknown primary: literature review. Ear Nose Throat J. 2014;93(4-5):E110.

13. Calabrese L, Jereczek-Fossa BA, Jassem J, et al. Diagnosis and management of neck metastases from an unknown primary. Acta Otorhinolaryngol Ital. 2005;25(1):2-12.

14. Davidson BJ, Spiro RH, Patel S, Patel K, Shah JP. Cervical metastases of occult origin: the impact of combined modality therapy. Am J Surg. 1994;168(5):395-9.

15. Al-Ibraheem A, Buck A, Krause BJ, Scheidhauer K, Schwaiger M. Clinical applications of FDG PET and PET/ CT in head and neck cancer. J Oncol. 2009;2009.

16. Diaconu C, Balaceanu A, Ghinescu M. A neck mass that disapears at compression: is it a reason for concern? Acta Medica Mediterranea. 2015;31(2):339-341.

17. Layfield LJ. Fine-needle aspiration in the diagnosis of head and neck lesions: A review and discussion of problems in differential diagnosis. Diagn Cytopathol. 2007;35(12):798-805.

18. Macdonald MR, Freeman JL, Hui MF, et al. Role of Epstein-Barr virus in fine-needle aspirates of metastatic neck nodes in the diagnosis of nasopharyngeal carcinoma. Head Neck. 1995;17(6):487-93.

19. Morris-Stiff G, Cheang P, Key S, Verghese A, Havard TJ. Does the surgeon still have a role to play in the diagnosis and management of lymphomas? World J Surg Oncol. 2008;6(1):13.

20. Urquhart A, Berg R. Hodgkin's and non-Hodgkin's lymphoma of the head and neck. Laryngoscope. 2001;111(9):1565-9.

21. Bozza F, Vigili MG, Ruscito P, Marzetti A, Marzetti F. Surgical management of parapharyngeal space tumours: results of 10-year follow-up. Acta Otorhinolaryngol Ital. 2009;29(1):10-5

22. Som PM, Biller HF, Lawson W. Tumors of the parapharyngeal space preoperative evaluation, diagnosis and surgical approaches. Ann Otol Rhinol Laryngol. 1981;90:3-15.

23. Sataloff RT. Sataloff's Comprehensive Textbook of Otolaryngology: Head \& Neck Surgery: Pediatric Otolaryngology. JP Medical Ltd; 2015;6.

24. Miller FR, Wanamaker JR, Lavertu P, Wood BG. Magnetic resonance imaging and the management of parapharyngeal space tumors. Head Neck J Sci Spec Head Neck. 1996;18(1):6777.

25. Storck K, Brandstetter M, Keller U, Knopf A. Clinical presentation and characteristics of lymphoma in the head and neck region. Head Face Med. 2019;15(1):1. 
26. Walter C, Ziebart T, Sagheb K, Rahimi-Nedjat RK, Manz A, Hess G. Malignant lymphomas in the head and neck region-a retrospective, single-center study over 41 years. Int J Med Sci. 2015;12(2):141-5

27. Savage SAH, Wotherspoon HA, Fitzsimons EJ, MacKenzie $\mathrm{K}$. Cervical lymphadenopathy resulting in a diagnosis of lymphoma. Scott Med J. 2008;53(3):13-6.

28. Dedivitis RA, Pfuetzenreiter Jr EG, de Castro MA Aspiration biopsy by fine needle of cervical adenopathy guided by ultrasonography. International Archives of Otorhynolaryngology. 2009;13(4):417-420.

29. Rzepakowska A, Zwierzyńska K, Osuch-Wójcikiewicz E, Niemczyk K. Lymphoid tissue neoplasms in the neck region - Epidemiological and clinical analysis over 15 years. Otolaryngol Pol. 2017;71(3):1-8.

30. Veness MJ, Morgan GJ, Palme CE, Gebski V. Surgery and adjuvant radiotherapy in patients with cutaneous head and neck squamous cell carcinoma metastatic to lymph nodes: combined treatment should be considered best practice. Laryngoscope. 2005;115(5):870-5.

31. Harrison LB, Sessions RB, Hong WK. Head and neck cancer: a multidisciplinary approach. Lippincott Williams \& Wilkins; 2009.

32. Suzuki S, Yasunaga H, Matsui H, Fushimi K, Saito Y, Yamasoba T. Factors associated with neck hematoma after thyroidectomy: a retrospective analysis using a Japanese inpatient database. Hung. S-H, editor. Medicine (Baltimore). 2016;95(7):e2812.

33. To VSH, Chan JYW, Tsang RKY, Wei WI. Review of salivary gland neoplasms. ISRN Otolaryngol. 2012;2012:872982.

34. Spiro RH. Management of malignant tumors of the salivary glands. Oncology (Williston Park). 1998;12(5):671-80.

35. Spiegel JH, Brys AK, Bhakti A, Singer MI. Metastasis to the submandibular gland in head and neck carcinomas. Head Neck. 2004;26(12):1064-8. 